\title{
Quasistatische ebene Stöße von Kugeln
}

Nach der Behandlung des reinen Normalstoßproblems ist das folgende Kapitel dem allgemeinen Stoßproblem von Kugeln gewidmet. Im Rahmen der im zweiten und dritten Kapitel beschriebenen Annahmen ist diese Aufgabe äquivalent zu dem ebenen Stoß einer starren Kugel auf einen deformierbaren Halbraum (siehe Abb.2.2). Unabhängig von der konkreten kontaktmechanischen Wechselwirkung ist die tangentiale Bewegungsgleichung wegen Gl. (2.27), unter Verwendung von Gl.(2.42), durch

$$
F_{x}=-\tilde{m} \kappa \ddot{u}_{x, K}
$$

gegeben. Hier bezeichnet $u_{x, K}$ die (makroskopische) tangentiale Verschiebung des Kontaktpunktes. Die Aufgabe besteht nun in der Formulierung eines exakten Kraftgesetzes für die Reibkraft $F_{x}$ und der mathematischen Lösung der sich ergebenden Differenzialgleichung. Das Stoßproblem wird durch die Angabe der tangentialen Stoßzahl (für die tangentiale Bewegung des Kontaktpunktes), $\epsilon_{x}$, gelöst.

\subsection{Elastischer schiefer Stoß ohne Gleiten}

Für den Grenzfall eines unendlich großen Reibbeiwertes (also der Abwesenheit lokalen Gleitens) gibt es in Lehrbüchern zu den Grundlagen der Technischen Mechanik eine scheinbar triviale Lösung des Stoßproblems [1, S. $171 \mathrm{f}$.]: Wegen der Haftbedingung verschwindet die tangentiale Geschwindigkeit des Kontaktpunktes, die Stoßzahl ist dann ganz einfach $\epsilon_{x}=0$. So simpel ist das Problem allerdings nicht. Aufgrund der Elastizität der Körper kann es auch ohne Gleiten eine tangentiale Bewegung im Kontakt geben; die Lösung mithilfe der globalen Haftbedingung für starre Körper ist daher in sich widersprüchlich. Eine widerspruchsfreie analytische Lösung für den Grenzfall ohne Gleiten wurde für elastisch homogene Medien von Barber [2] und Jäger [3] präsentiert. Diese Lösung kann man ohne Schwierigkeiten für Medien mit einer elastischen Gradierung in der Form eines Potenzgesetzes verallgemeinern. 


\subsubsection{Homogene Medien}

Wegen des wachsenden, bzw. schrumpfenden Kontaktradius ist das kontaktmechanische Verhalten während der Kompressions- und der Restitutionsphase qualitativ unterschiedlich. Beide Teile des Stoßes müssen daher getrennt voneinander behandelt werden.

\section{Kompressionsphase}

Während der Kompressionsphase kann man das Kraftgesetz inkrementell formulieren. Da zu keinem Zeitpunkt lokales Gleiten zugelassen ist, wird zu einem Zeitpunkt $t$ das Kontaktgebiet mit dem Radius $a(t)$ als Ganzes um $\mathrm{d} u_{x, K}(t)$ verschoben. Der entsprechende Beitrag zu der tangentialen Kraft ist wegen Gl.(3.92)

$$
d F_{x}=2 \tilde{G} a \mathrm{~d} u_{x, K}
$$

Division durch $\mathrm{d} t$ und Einsetzen der Gl. (6.1) sowie der Relation (3.35) für den Kontaktradius ergibt

$$
\frac{\mathrm{d}^{3} u_{x, K}}{\mathrm{~d} t^{3}}+\frac{2 \tilde{G}}{\kappa \tilde{m}} \sqrt{\tilde{R} d} \frac{\mathrm{d} u_{x, K}}{\mathrm{~d} t}=0 .
$$

Führt man die dimensionsfreien Größen

$$
\hat{t}:=\frac{\left|v_{z, K, 0}\right|}{d_{\max }} t, \quad \hat{u}_{x, K}:=\frac{u_{x, K}}{d_{\max }}, \quad \hat{v}_{x, K}:=\frac{\mathrm{d} \hat{u}_{x, K}}{\mathrm{~d} \hat{t}},
$$

mit der maximalen Eindrucktiefe $d_{\max }$ aus Gl.(5.8), ein, erhält man

$$
\frac{\mathrm{d}^{2} \hat{v}_{x, K}}{\mathrm{~d} \hat{t}^{2}}+\frac{15}{4} \chi \sqrt{\frac{d}{d_{\max }}} \hat{v}_{x, K}=0, \quad \chi:=\frac{l}{2 \kappa}, \quad l:=\frac{\tilde{G}}{\tilde{E}}=\frac{2-2 v}{2-v},
$$

was mithilfe der sich aus Gl.(5.10) ergebenden Beziehung

$$
\frac{\mathrm{d} \xi}{\mathrm{d} \hat{t}}=\frac{5}{2} \xi^{3 / 5}(1-\xi)^{1 / 2}, \quad \xi:=\left(\frac{d}{d_{\max }}\right)^{5 / 2},
$$

und der Kettenregel in die Hypergeometrische Gleichung

$$
\frac{\mathrm{d}^{2} \hat{v}_{x, K}}{\mathrm{~d} \xi^{2}} \xi(1-\xi)+\frac{1}{10}(6-11 \xi) \frac{\mathrm{d} \hat{v}_{x, K}}{\mathrm{~d} \xi}+\frac{3 \chi}{5} \hat{v}_{x, K}=0
$$

überführt werden kann. Den Parameter $\chi$ führten Maw et al. [4] zur Beschreibung schiefer elastischer Stöße von Kugeln ein. Der darin enthaltene Parameter $l$ gibt das Verhältnis der tangentialen zur normalen Steifigkeit des Flachstempel-Kontaktes an und wird häufig nach Mindlin benannt. In der Umgebung von $\xi=0$ hat Gl.(6.7) die allgemeine Lösung (siehe Gl. (9.37) im Anhang) 


$$
\begin{aligned}
\hat{v}_{x, K}(\xi)= & C_{12} \mathrm{~F}_{1}\left(\frac{1+\phi}{20}, \frac{1-\phi}{20} ; \frac{3}{5} ; \xi\right) \\
& +C_{2} \xi^{2 / 5}{ }_{2} \mathrm{~F}_{1}\left(\frac{9+\phi}{20}, \frac{9-\phi}{20} ; \frac{7}{5} ; \xi\right), \quad \phi:=\sqrt{1+240 \chi}
\end{aligned}
$$

mit der ebenfalls im Anhang gegebenen Hypergeometrischen Funktion ${ }_{2} \mathrm{~F}_{1}$. Die Integrationskonstanten $C_{1}$ und $C_{2}$ ergeben sich aus den Anfangs-, bzw. Randbedingungen:

$$
\hat{v}_{x, K}(\xi=0)=C_{1}=\tan \alpha, \quad \frac{\mathrm{d} \hat{v}_{x, K}}{\mathrm{~d} \hat{t}}(\xi=0)=\frac{5}{2} C_{2}=0,
$$

wobei der Einfallswinkel $\alpha$ der Bewegung des Kontaktpunktes in Gl. (2.58) definiert wurde. Am Umkehrpunkt ergibt sich damit die Geschwindigkeit

$$
\hat{v}_{x, K}(\xi=1)=\tan \alpha_{2} \mathrm{~F}_{1}\left(\frac{1+\phi}{20}, \frac{1-\phi}{20} ; \frac{3}{5} ; 1\right)=\tan \alpha \frac{\sqrt{\pi} \Gamma(3 / 5)}{\Gamma\left(\frac{11+\phi}{20}\right) \Gamma\left(\frac{11-\phi}{20}\right)} .
$$

Die Tangentialkraft ergibt sich aus Gl.(6.1) mithilfe von Gl.(6.6) und der im Anhang gegebenen Beziehung (9.40) zu

$$
F_{x}(\xi)=l \tan \alpha F_{N, \max } \xi^{3 / 5}{ }_{2} \mathrm{~F}_{1}\left(\frac{11+\phi}{20}, \frac{11-\phi}{20} ; \frac{8}{5} ; \xi\right),
$$

mit der maximalen Normaldruckkraft $F_{N \text {, max }}$ aus Gl.(5.9).

\section{Restitutionsphase}

Während der Restitutionsphase kehrt sich das Vorzeichen der Normalgeschwindigkeit um, sodass Gl.(6.6) durch

$$
\frac{\mathrm{d} \xi}{\mathrm{d} \hat{t}}=-\frac{5}{2} \xi^{3 / 5}(1-\xi)^{1 / 2}
$$

ersetzt werden muss. Außerdem gibt es für jeden Zeitpunkt ${ }^{1} t>T_{S} / 2$ einen Zeitpunkt $t_{c}(t)=T_{S}-t$ während der Kompressionsphase, sodass $a(t)=a\left(t_{c}\right)$. Da zu keinem Zeitpunkt lokales Gleiten zugelassen wird, ist die Differenz der Tangentialkräfte zwischen diesen beiden Zeitpunkten durch die erfolgte tangentiale Verschiebung des Kontaktgebiets mit dem Radius $a(t)$ als Ganzes vorgegeben:

$$
F_{x}(t)-F_{x}\left(t_{c}\right)=2 \tilde{G} a(t)\left[u_{K, x}(t)-u_{K, x}\left(t_{c}\right)\right] .
$$

Da weiterhin die Bewegungsgleichung (6.1) sowohl für die Kompressions- als auch für die Restitutionsphase gültig ist, ist die Bewegungsgleichung für die Differenz der Verschiebung,

$$
u_{x, K}^{*}(t):=u_{x, K}(t)-u_{x, K}\left(t_{c}\right)
$$

${ }^{1} T_{S}$ ist die in Gl.(5.12) gegebene Stoßdauer. 
durch

$$
\frac{\mathrm{d}^{2} u_{x, K}^{*}}{\mathrm{~d} t^{2}}+\frac{2 \tilde{G}}{\kappa \tilde{m}} \sqrt{\tilde{R} d} u_{x, K}^{*}=0 .
$$

gegeben. Die Größe $u_{x, K}^{*}$ muss also der gleichen Hypergeometrischen Differenzialgleichung (6.7) genügen wie $\dot{u}_{x, K}$ während der Kompressionsphase. Die allgemeine Lösung dieser Gleichung in der Umgebung von $\xi=1$ (d.h. dem Umkehrpunkt der Normalbewegung) ist (siehe Gl. (9.38) im Anhang)

$$
\begin{aligned}
\frac{u_{x, K}^{*}(\xi)}{d_{\max }}= & C_{3{ }_{2} \mathrm{~F}_{1}}\left(\frac{1+\phi}{20}, \frac{1-\phi}{20} ; \frac{1}{2} ; 1-\xi\right) \\
& +C_{4} \sqrt{1-\xi}{ }_{2} \mathrm{~F}_{1}\left(\frac{11+\phi}{20}, \frac{11-\phi}{20} ; \frac{3}{2} ; 1-\xi\right)
\end{aligned}
$$

Dabei ist offenbar

$$
\frac{u_{x, K}^{*}(\xi=1)}{d_{\max }}=C_{3}=0 .
$$

Mit den in den Gl. (9.40) und (9.41) gegebenen Beziehungen und der Relation (6.12) erhält man für die Geschwindigkeit

$$
\begin{aligned}
\hat{v}_{x, K}(\xi)= & -\tan \alpha_{2} \mathrm{~F}_{1}\left(\frac{1+\phi}{20}, \frac{1-\phi}{20} ; \frac{3}{5} ; \xi\right) \\
& +\frac{5}{4} C_{4}{ }_{2} \mathrm{~F}_{1}\left(\frac{-1+\phi}{20}, \frac{-1-\phi}{20} ; \frac{1}{2} ; 1-\xi\right)
\end{aligned}
$$

Die Integrationskonstante $C_{4}$ ergibt sich wegen der Stetigkeit der Geschwindigkeit aus Gl. (6.10) zu

$$
\frac{5}{4} C_{4}=2 \tan \alpha_{2} \mathrm{~F}_{1}\left(\frac{1+\phi}{20}, \frac{1-\phi}{20} ; \frac{3}{5} ; 1\right) .
$$

Die tangentiale Stoßzahl beträgt dann unter Berücksichtigung der Gl.(9.39) im Anhang

$$
\epsilon_{x}=1-2 \pi\left[\frac{\Gamma(3 / 5)}{\Gamma\left(\frac{11+\phi}{20}\right) \Gamma\left(\frac{11-\phi}{20}\right)}\right]^{2} .
$$

Die Gamma-Funktion ist für alle negativen ganzen Zahlen (einschließlich Null) singulär.

Die Stoßzahl ist an den singulären Punkten, die entsprechend durch die Bedingung

$$
\chi_{c}=\frac{1+2 i}{2}\left(1+\frac{5 i}{3}\right), \quad i \in \mathbb{N},
$$


Abb.6.1 Tangentiale Stoßzahl für den elastischen Stoß ohne Gleiten als Funktion des Parameters $\chi$. Die durchgezogene Kurve bezeichnet die analytische Lösung 6.20 von Jäger [3], die Kreise markieren die Ergebnisse einer numerischen Lösung des Problems mithilfe der MDR

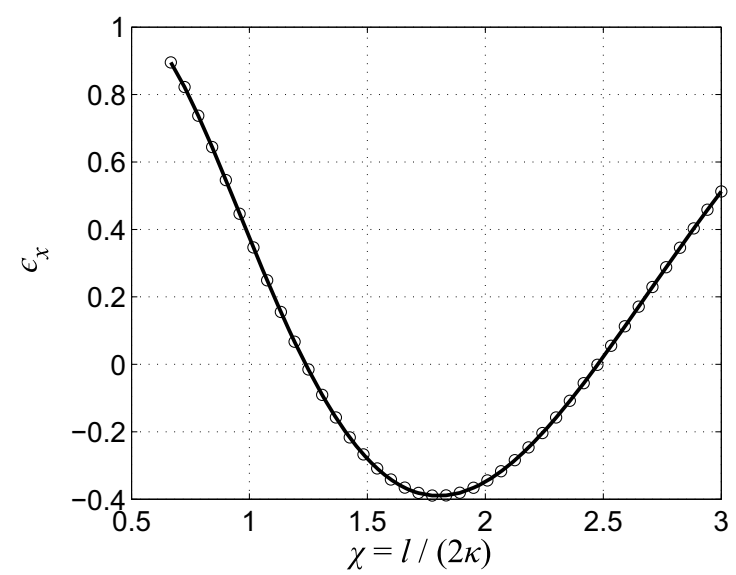

gegeben sind, gleich Eins, während des Stoßes wird dann keine Energie dissipiert ${ }^{2}$. Das analytische Ergebnis (6.20) ist in Abb. 6.1 gemeinsam mit einer auf der MDR beruhenden numerischen Lösung gezeigt, die von Lyashenko und Popov [5] publiziert wurde.

Für die Tangentialkraft während der Restitutionsphase ergibt sich wegen Gl.(6.13) und mit den im Anhang gegebenen Vereinfachungen der Hypergeometrischen Funktion

$$
F_{x}(\xi)=F_{x}^{\mathrm{k}}+\frac{12}{5} F_{N, \max } \frac{\sqrt{\pi} \Gamma(3 / 5) l \tan \alpha}{\Gamma\left(\frac{11+\phi}{20}\right) \Gamma\left(\frac{11-\phi}{20}\right)} \xi^{1 / 5} \sqrt{1-\xi}{ }_{2} \mathrm{~F}_{1}\left(\frac{11+\phi}{20}, \frac{11-\phi}{20} ; \frac{3}{2} ; 1-\xi\right) .
$$

Dabei muss für $F_{x}^{\mathrm{k}}$ der Ausdruck (6.11) aus der Kompressionsphase eingesetzt werden. Die Tangentialkraft, normiert auf $l \tan \alpha F_{N \text {,max }}$, als Funktion der Zeit, normiert auf $T_{S}$, hängt damit nur von dem Parameter $\chi$ ab. Der Zeitverlauf der normierten Tangentialkraft während des ganzen Stoßvorgangs ist in Abb. 6.2 für verschiedene Werte von $\chi$ gezeigt. Für den singulären Fall $\chi=4$ ist der Verlauf symmetrisch, die Kraftstöße während der Kompressions- und der Restututionsphase sind daher gleich. Dies trifft für alle in Gl. (6.21) definierten singulären Fälle zu, nur steigt mit $i$ die Anzahl der Minima und Maxima des Kraftverlaufes [3].

\section{Nicht-parabolische Indenterformen}

Die oben gezeigte Lösung für parabolische Kontakte kann für Indenterprofile in der Form des Potenzgesetzes (3.38) verallgemeinert werden, wenn man annimmt, dass die Bewegungsgleichung (6.1) - die aus der makroskopischen Dynamik zusammenstoßender Kugeln

\footnotetext{
${ }^{2}$ Dabei muss man bedenken, dass die physikalische Untergrenze für $\chi$ bei elastisch homogenen Kugeln durch den Wert 2/3 gegeben ist, der erste singuläre Fall entspricht dann $\chi=4$.
} 
Abb.6.2 Normierte

Tangentialkraft als Funktion der normierten Zeit für den elastischen Stoß ohne Gleiten bei verschiedenen Werten des Parameter $\chi$

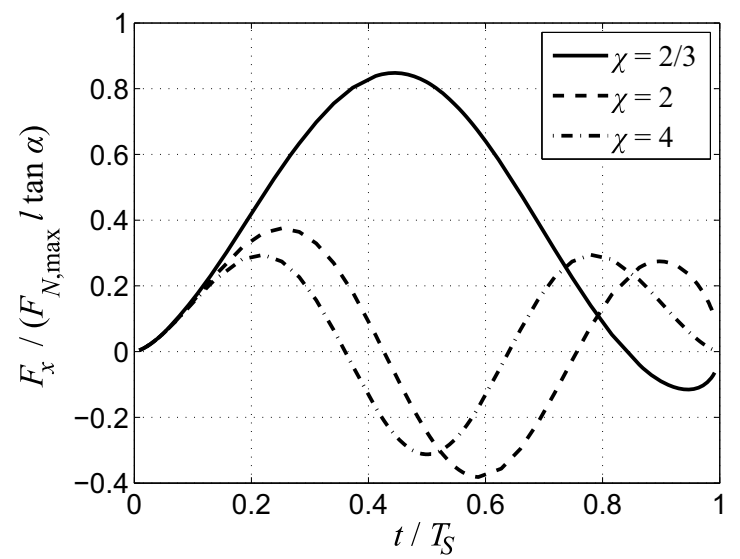

hergeleitet wurde - trotzdem ihre Gültigkeit behält ${ }^{3}$. Aus der Lösung des Normalstoßproblems erhält man, dass der Zusammenhang (6.6) während der Kompressionsphase durch

$$
\frac{\mathrm{d} \xi}{\mathrm{d} \hat{t}}=\frac{2 n+1}{n} \xi^{\frac{n+1}{2 n+1}}(1-\xi)^{1 / 2}, \quad \xi:=\left(\frac{d}{d_{\max }}\right)^{\frac{2 n+1}{n}}
$$

ersetzt werden muss. Mit dem aus der Lösung des Kontaktproblems bekannten Zusammenhang zwischen Kontaktradius und Eindrucktiefe, siehe Gl.(3.39), erhält man während der Kompressionsphase die Gleichung

$$
\frac{\mathrm{d}^{2} \hat{v}_{x, K}}{\mathrm{~d} \xi^{2}} \xi(1-\xi)+\frac{2 n+2-(4 n+3) \xi}{2(2 n+1)} \frac{\mathrm{d} \hat{v}_{x, K}}{\mathrm{~d} \xi}+\frac{n+1}{2 n+1} \chi \hat{v}_{x, K}=0
$$

mit der Lösung

$\hat{v}_{x, K}(\xi)=\tan \alpha_{2} \mathrm{~F}_{1}\left(\frac{1+\phi}{8 n+4}, \frac{1-\phi}{8 n+4} ; \frac{n+1}{2 n+1} ; \xi\right), \quad \phi:=\sqrt{1+16\left(2 n^{2}+3 n+1\right) \chi}$,

für die normierte tangentiale Geschwindigkeit. Der Parameter $\chi$ ist dabei unverändert durch die in Gl. (6.5) gegebene Definition bestimmt. Die Tangentialkraft während der Kompressionsphase ergibt sich zu

$$
F_{x}(\xi)=l \tan \alpha F_{N, \max } \xi^{\frac{n+1}{2 n+1}}{ }_{2} \mathrm{~F}_{1}\left(\frac{4 n+3+\phi}{8 n+4}, \frac{4 n+3-\phi}{8 n+4} ; \frac{3 n+2}{2 n+1} ; \xi\right) .
$$

\footnotetext{
${ }^{3}$ Dies kann beispielsweise der Fall sein, wenn die makroskopische Form der zusammenstoßenden Körper in guter Näherung Kugeln entspricht, aber die Profilform in der Nähe des Kontaktpunktes von der parabolischen stark abweicht.
} 
Die normierte Geschwindigkeit während der Restitutionsphase beträgt

$$
\begin{aligned}
\hat{v}_{x, K}(\xi)= & -\tan \alpha_{2} \mathrm{~F}_{1}\left(\frac{1+\phi}{8 n+4}, \frac{1-\phi}{8 n+4} ; \frac{n+1}{2 n+1} ; \xi\right) \\
& +\frac{2 n+1}{2 n} C_{4}{ }_{2} \mathrm{~F}_{1}\left(\frac{-1+\phi}{8 n+4}, \frac{-1-\phi}{8 n+4} ; \frac{1}{2} ; 1-\xi\right),
\end{aligned}
$$

mit

$$
\frac{2 n+1}{2 n} C_{4}=2 \tan \alpha_{2} \mathrm{~F}_{1}\left(\frac{1+\phi}{8 n+4}, \frac{1-\phi}{8 n+4} ; \frac{n+1}{2 n+1} ; 1\right),
$$

und für die Tangentialkraft während der Restitutionsphase erhält man

$$
\begin{aligned}
F_{x}(\xi)= & F_{x}^{\mathrm{k}}+\frac{4(n+1)}{2 n+1} F_{N, \max } \frac{\sqrt{\pi} \Gamma\left(\frac{n+1}{2 n+1}\right) l \tan \alpha}{\Gamma\left(\frac{4 n+3+\phi}{8 n+4}\right) \Gamma\left(\frac{4 n+3-\phi}{8 n+4}\right)} \xi^{\frac{1}{2 n+1}} \sqrt{1-\xi} \\
& \times{ }_{2} \mathrm{~F}_{1}\left(\frac{4 n+3+\phi}{n+4}, \frac{4 n+3-\phi}{8 n+4} ; \frac{3}{2} ; 1-\xi\right) .
\end{aligned}
$$

Die Stoßzahl

$$
\epsilon_{x}=1-2 \pi\left[\frac{\Gamma\left(\frac{n+1}{2 n+1}\right)}{\Gamma\left(\frac{4 n+3+\phi}{8 n+4}\right) \Gamma\left(\frac{4 n+3-\phi}{8 n+4}\right)}\right]^{2}
$$

mit den singulären Punkten der Gamma-Funktion,

$$
\chi_{c}=\frac{1+2 i}{2(n+1)}(1+i+n+2 i n), \quad i \in \mathbb{N},
$$

ist in Abb. 6.3 als Konturlinien-Diagramm dargestellt. Für $n \rightarrow \infty$ ergibt sich der Flachstempelkontakt und daher das Ergebnis, wenn der Kontakt durch eine einzelne lineare Feder approximiert wird [5],

$$
\lim _{n \rightarrow \infty} \epsilon_{x}=-\cos (\pi \sqrt{2 \chi})
$$

\subsubsection{Funktionale Gradientenmedien}

Analog zur Herleitung aus dem vorherigen Abschnitt kann man auch den ebenen Stoß ohne Gleiten für Medien mit einer elastischen Gradierung in der Form eines Potenzgesetzes, siehe Gl.(3.209), vollständig analytisch lösen. Es werden zunächst kollidierende Kugeln betrachtet. Dabei muss man wiederum die Kompressions- und Restitutionsphase getrennt voneinander behandeln. 
Abb.6.3 KonturlinienDiagramm der tangentialen Stoßzahl (6.30) für den elastischen Stoß ohne Gleiten mit einem Potenzprofil als Funktion der Parameter $\chi$ und $n$. Die gestrichelten Linien bezeichnen die singulären Punkte nach Gl.(6.31), für die während des Stoßes keine Energie dissipiert wird

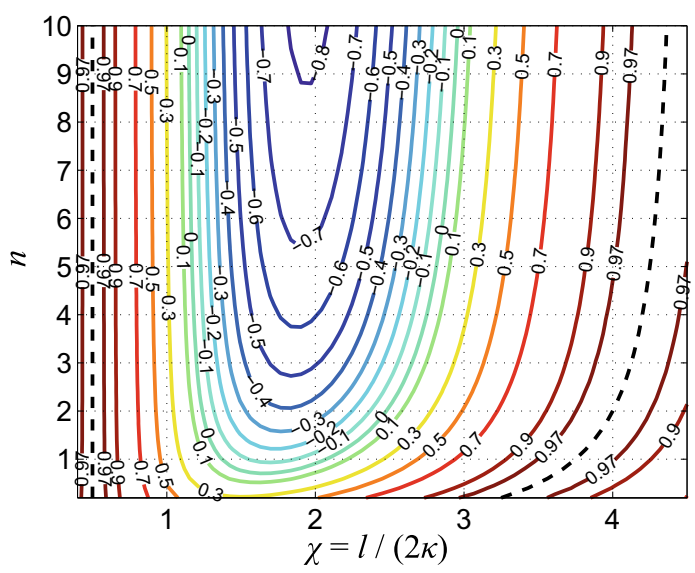

\section{Kompressionsphase}

Der inkrementelle Beitrag zur Tangentialkraft ist wegen Gl.(3.254) nun durch

$$
d F_{x}=\frac{2}{1+k} c_{T} a^{1+k} \mathrm{~d} u_{x, K} .
$$

gegeben. Mit der Lösung des Normalproblems und den gleichen Normierungen wie in Gl. (6.4), wobei für die maximale Eindrucktiefe der Wert aus Gl. (5.21) verwendet werden muss, erhält man die dimensionsfreie Bewegungsgleichung in tangentialer Richtung,

$$
\frac{\mathrm{d}^{2} \hat{v}_{x, K}}{\mathrm{~d} \hat{t}^{2}}+\frac{(3+k)(5+k)}{4} \chi\left(\frac{d}{d_{\max }}\right)^{\frac{1+k}{2}} \hat{v}_{x, K}=0, \quad \chi:=\frac{l}{2 \kappa}, \quad l:=\frac{c_{T}}{c_{N}} .
$$

Die Größe $\chi$ ist hier die Generalisierung des von Maw et al. [4] vorgeschlagenen Parameters für Gradientenmedien mit einer Gradierung in der Form eines Potenzgesetzes. Die Moduln $c_{N}$ und $c_{T}$ sind in den Gl.(3.223) und (3.224) gegeben. Mit der Substitution

$$
\xi:=\left(\frac{d}{d_{\max }}\right)^{\frac{5+k}{2}}
$$

und der sich aus der Energieerhaltung (5.20) ergebenden Beziehung

$$
\frac{\mathrm{d} \xi}{\mathrm{d} \hat{t}}=\frac{5+k}{2} \xi^{\frac{3+k}{5+k}}(1-\xi)^{1 / 2}
$$

kann man die Bewegungsgleichung, analog zum homogenen Fall, in die Hypergeometrische Differenzialgleichung

$$
\frac{\mathrm{d}^{2} \hat{v}_{x, K}}{\mathrm{~d} \xi^{2}} \xi(1-\xi)+\frac{6+2 k-(11+3 k) \xi}{10+2 k} \frac{\mathrm{d} \hat{v}_{x, K}}{\mathrm{~d} \xi}+\frac{3+k}{5+k} \chi \hat{v}_{x, K}=0
$$


überführen. Diese hat unter Berücksichtigung der Anfangsbedingungen in der Umgebung von $\xi=0$ die Lösung

$$
\hat{v}_{x, K}(\xi)=\tan \alpha_{2} \mathrm{~F}_{1}\left(\frac{1+k+\phi}{20+4 k}, \frac{1+k-\phi}{20+4 k} ; \frac{3+k}{5+k} ; \xi\right), \quad \phi:=\sqrt{(1+k)^{2}+16(3+k)(5+k) \chi} .
$$

Die Tangentialkraft ergibt sich zu

$$
F_{x}(\xi)=l \tan \alpha F_{N, \max } \xi^{\frac{3+k}{5+k}}{ }_{2} \mathrm{~F}_{1}\left(\frac{11+3 k+\phi}{20+4 k}, \frac{11+3 k-\phi}{20+4 k} ; \frac{8+2 k}{5+k} ; \xi\right),
$$

mit der aus der Lösung des Normalproblems bekannten maximalen Normalkraft und dem in Gl. (2.58) definierten Stoßwinkel.

\section{Restitutionsphase}

Die Behandlung der Restitutionsphase ist ebenfalls völlig analog zum speziellen homogenen Fall. Aus Platzgründen soll daher an dieser Stelle auf die Darstellung der Herleitung verzichtet werden. Man erhält für die Differenz der tangentialen Verschiebung

$$
\frac{u_{x, K}^{*}(\xi)}{d_{\max }}=C_{4} \sqrt{1-\xi}{ }_{2} \mathrm{~F}_{1}\left(\frac{11+3 k+\phi}{20+4 k}, \frac{11+3 k-\phi}{20+4 k} ; \frac{3}{2} ; 1-\xi\right),
$$

mit

$$
C_{4}=\frac{8}{5+k} \tan \alpha_{2} \mathrm{~F}_{1}\left(\frac{1+k+\phi}{20+4 k}, \frac{1+k-\phi}{20+4 k} ; \frac{3+k}{5+k} ; 1\right),
$$

für die normierte tangentiale Geschwindigkeit

$$
\begin{aligned}
\hat{v}_{x, K}(\xi)= & -\tan \alpha_{2} \mathrm{~F}_{1}\left(\frac{1+k+\phi}{20+4 k}, \frac{1+k-\phi}{20+4 k} ; \frac{3+k}{5+k} ; \xi\right) \\
& +\frac{5+k}{4} C_{4}{ }_{2} \mathrm{~F}_{1}\left(\frac{-1-k+\phi}{20+4 k}, \frac{-1-k-\phi}{20+4 k} ; \frac{1}{2} ; 1-\xi\right),
\end{aligned}
$$

die Tangentialkraft

$$
F_{x}(\xi)=F_{x}^{\mathrm{k}}+\frac{3+k}{2} l F_{N, \max } C_{4} \xi^{\frac{1+k}{5+k}} \sqrt{1-\xi_{2}} \mathrm{~F}_{1}\left(\frac{11+3 k+\phi}{20+4 k}, \frac{11+3 k-\phi}{20+4 k} ; \frac{3}{2} ; 1-\xi\right)
$$

und die tangentiale Stoßzahl

$$
\epsilon_{x}=1-2 \pi\left[\frac{\Gamma\left(\frac{3+k}{5+k}\right)}{\Gamma\left(\frac{11+3 k+\phi}{20+4 k}\right) \Gamma\left(\frac{11+3 k-\phi}{20+4 k}\right)}\right]^{2} .
$$


Abb.6.4 KonturlinienDiagramm der tangentialen Stoßzahl (6.44) für den elastischen Stoß auf ein Gradientenmedium ohne Gleiten als Funktion der Parameter $\chi$ und $k$. Die gestrichelten Linien bezeichnen die singulären Punkte nach Gl.(6.45)

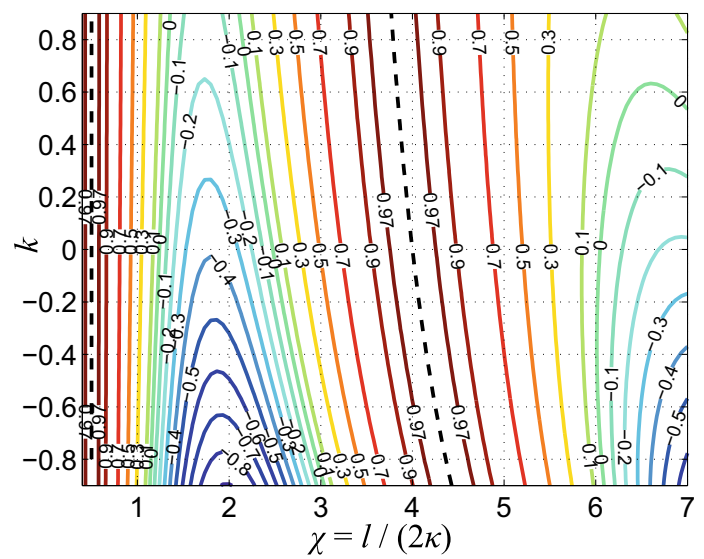

Die singulären Punkte der Gamma-Funktion, für die die Stoßzahl Eins ist, liegen bei

$$
\chi_{c}=\frac{1+2 i}{2}\left(1+i \frac{5+k}{3+k}\right), \quad i \in \mathbb{N} .
$$

Die tangentiale Stoßzahl als Funktion der beiden definierenden Parameter $\chi$ und $k$ ist als Konturlinien-Diagramm in Abb.6.4 gezeigt. Da das Mindlin-Verhältnis $l$ für Gradientenmedien Werte aus einem deutlich breiteren Bereich annehmen kann als im homogenen Fall (siehe Abb.3.13), wurde für die Darstellung ein größerer Definitionsbereich für $\chi$ gewählt als in Abb. 6.1.

\section{Nicht-parabolische Indenterformen}

Wenn man wiederum das tangentiale Stoßproblem ohne Gleiten für einen Indenter mit einem Profil in der Nähe des Kontaktes in der Form eines Potenzgesetzes mit dem Exponenten $n$ untersucht (unter den gleichen Annahmen wie weiter oben für homogene Medien), stellt man wie im Fall des reinen Normalstoßes fest, dass das inhomogene Problem in normierten Größen auf das homogene Problem mit dem in Gl. (3.241) eingeführten „,korrigierten“ Exponenten des Profils zurückgeführt werden kann. Insbesondere kann man die in den Abb. 6.3 und 6.4 gezeigten Verläufe für die tangentiale Stoßzahl durch die Koordinatentransformation $n=2 /(1+k)$ ineinander überführen.

\subsection{Viskoelastischer schiefer Stoß ohne Gleiten}

Wenn das Stoß-Kontakt-Problem für den Normalstoß einer starren Kugel auf ein viskoelastisches Medium numerisch mithilfe der MDR gelöst wurde (siehe das Unterkapitel 5.4), stellt die Behandlung der ebenen Kollision ohne Gleiten keine Schwierigkeiten dar: Das Kontaktgebiet mit dem Radius $a$ - der Verlauf von $a(t)$ ist dabei aus der Lösung des 
Normalproblems bekannt - wird zu jedem Zeitpunkt durch die tangentiale Bewegung $u_{x, K}$ des „Kontaktpunktes“ auf der starren Kugel tangential verschoben. Daraus ergibt sich im MDR-Modell in jedem Zeitschritt die Streckenlast $q_{x}$ der viskoelastischen Bettung und damit die gesamte Tangentialkraft $F_{x}$, mit der man die Bewegungsgleichung (6.1) in diskreten Zeitschritten lösen kann. Im Folgenden werden als rheologische Modelle der KelvinVoigt-Körper und der Kelvin-Maxwell-Körper berücksichtigt. Willert et al. [6] berücksichtigten außerdem das Standard-Medium für das ebene viskoelastische Stoßproblem.

\subsubsection{Inkompressibles Kelvin-Voigt-Medium}

Im Fall des Kelvin-Voigt-Mediums entkoppeln die elastischen und viskosen Eigenschaften (charakterisiert durch den Schubmodul $G$ sowie die Scherviskosität $\eta$ ) und man kann daher die Streckenlast in einen elastischen und viskosen Anteil aufteilen:

$$
q_{x}(x, t)=q_{x}^{\mathrm{el}}(x, t)+q_{x}^{\mathrm{vis}}(x, t) .
$$

Der viskose Anteil ist einfach

$$
q_{x}^{\mathrm{vis}}(x, t)=\frac{8}{3} \eta \dot{u}_{x, K}, \quad|x| \leq a(t),
$$

und der elastische Anteil wird inkrementell in der Form

$$
\mathrm{d} q_{x}^{\mathrm{el}}(x, t)=\frac{8}{3} G \mathrm{~d} u_{x, K}, \quad|x| \leq a(t),
$$

ausgewertet. Integration der Streckenlast über das Kontaktgebiet und Auswertung der Bewegungsgleichung (6.1) liefern die tangentiale Bewegung des Kontaktpunktes und damit die tangentiale Stoßzahl $\epsilon_{x}$. Durch Dimensionsanalyse und numerische Studien kann man leicht feststellen, dass diese nur von den beiden dimensionslosen Parametern

$$
\delta:=\eta\left(\frac{\tilde{R} v_{z, K, 0}}{\tilde{m}^{2} G^{3}}\right)^{1 / 5}, \quad \chi:=\frac{l}{2 \kappa}=\frac{1}{3 \kappa}
$$

abhängt. Dabei wurde berücksichtigt, dass das Mindlin-Verhältnis $l$ wegen der Inkompressibilität durch den Wert $l=2 / 3$ gegeben ist. Bei der Behandlung des Normalstoßproblems wurde außerdem gezeigt, dass die normale Stoßzahl $\epsilon_{z}$ für den Kelvin-Voigt-Körper eine streng monoton fallende Funktion von $\delta$ ist, man kann daher die tangentiale Stoßzahl auch als Funktion von $\epsilon_{z}$ und $\chi$ darstellen. Diese Lösung des tangentialen Stoßproblems ist in Abb. 6.5 gezeigt. Für $\epsilon_{z}=1$ ergibt sich natürlich die elastische Lösung nach Gl. (6.20), für $\epsilon_{z} \rightarrow 0$ wird auch die tangentiale Bewegung vollständig gedämpft. 
Abb.6.5 KonturlinienDiagramm der tangentialen Stoßzahl für den ebenen Stoß ohne Gleiten einer Kugel auf ein inkompressibles Kelvin-Voigt-Medium als Funktion des Parameters $\chi$ und der normalen Stoßzahl

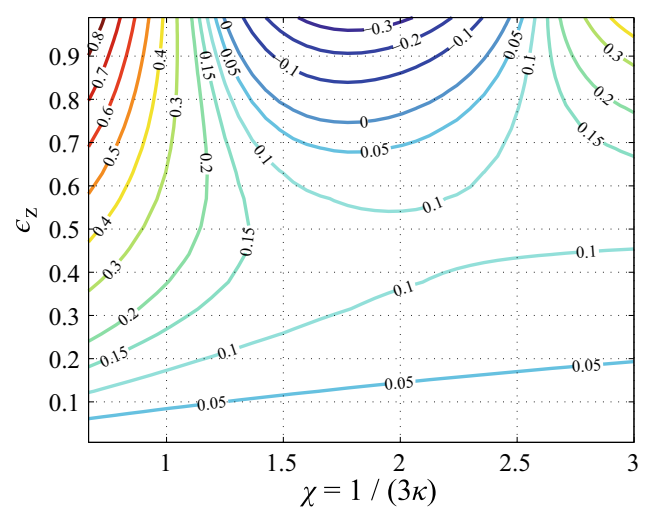

\subsubsection{Inkompressibles Kelvin-Maxwell-Medium}

Die Behandlung des Kelvin-Maxwell-Mediums bereitet ebenfalls keine Schwierigkeiten, wenn das dazugehörige Normalstoßproblem bereits gelöst wurde. Es sei angemerkt, dass im Folgenden alle Notationen aus dem Abschn. 5.4.4 übernommen werden.

In Analogie zur Bestimmung der normalen Streckenlast mittels Gl. (5.92) ist die tangentiale Streckenlast durch die Beziehung

$$
q_{x}(x, t)=\frac{8}{3}\left(G_{\infty} u_{x}(x, t)+G_{1} \tilde{u}_{x}(x, t)+\eta_{0} \dot{u}_{x}(x, t)\right)
$$

bestimmt. Hier bezeichnen $u_{x}$ und $\tilde{u}_{x}$ die tangentialen Verschiebungen des äußeren und inneren Knotens des Kelvin-Maxwell-Elements an der Stelle $x$. Wegen der Abwesenheit lokalen Gleitens ist $\dot{u}_{x}$ wiederum durch die Bewegung der Kugel vorgegeben und $u_{x}$ kann daher, wie im vorherigen Abschnitt, inkrementell bestimmt werden. Die Bewegung des inneren Freiheitsgrads $\tilde{u}_{x}$ ist durch das Gleichgewicht des inneren Knotens in tangentialer Richtung,

$$
0=\tilde{u}_{x}+\tau\left(\dot{\tilde{u}}_{x}-\dot{u}_{x}\right)
$$

festgelegt. Die tangentiale Bewegung des Kontaktpunktes auf der starren Kugel kann man wie bisher durch Integration von $q_{x}$ über das Kontaktgebiet und Lösung der Gl. (6.1) bestimmen.

Durch Dimensionsanalyse und numerische Studien lässt sich zeigen, dass die tangentiale Stoßzahl $\epsilon_{x}$ nur von den dimensionsfreien Parametern

$$
\beta:=\frac{G_{\infty}}{G_{1}}, \quad \delta:=\eta_{0}\left(\frac{\tilde{R} v_{z, K, 0}}{\tilde{m}^{2} G_{\infty}^{3}}\right)^{1 / 5}, \quad \chi:=\frac{1}{3 \kappa}
$$


Abb.6.6 KonturlinienDiagramm der tangentialen Stoßzahl für den ebenen Stoß ohne Gleiten einer Kugel auf ein inkompressibles

Kelvin-Maxwell-Medium als Funktion des Parameters $\chi$ und der normalen Stoßzahl für $G_{\infty} / G_{1}=1 / 100$

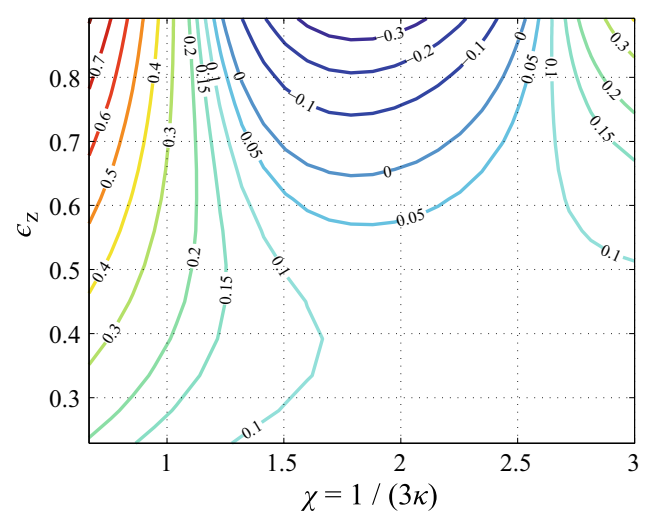

abhängt. Für jedes feste $\beta$ ist dabei die normale Stoßzahl eine streng monoton fallende Funktion von $\delta$ (siehe Abb. 5.14), eine alternative Parametrisierung besteht also in der Verwendung von $\epsilon_{z}, \beta$ und $\chi$.

Die Abb. 6.6 zeigt ein Konturlinien-Diagramm der tangentialen Stoßzahl als Funktion von $\epsilon_{z}$ und $\chi$ für $\beta=1 / 100$. Für diesen Wert von $\beta$ unterscheidet sich das Materialverhalten wesentlich von dem des Kelvin-Voigt-Mediums und trotzdem sind bei der Lösung des tangentialen Stoßproblems nur geringe Abweichungen von dem in Abb. 6.5 gezeigten Ergebnis für das Kelvin-Voigt-Material erkennbar. Das legt nahe, dass auch für den schiefen viskoelastischen Stoß die konkrete viskoelastische Rheologie nur eine untergeordnete Rolle spielt und daher zur Beschreibung die einfachste Rheologie, das Kelvin-Voigt-Medium, ausreicht, wenn zur Charakterisierung des Materialverhaltens die normale Stoßzahl - die, wie im letzten Kapitel gezeigt, wiederum hauptsächlich durch das Verhältnis des Verlust- und Speichermoduls auf der Zeitskala der Stoßdauer bestimmt ist - herangezogen wird.

\subsection{Elastischer schiefer Stoß mit Gleiten}

Die Annahme eines unendlich großen Reibungskoeffizienten ist natürlich eine grobe Vereinfachung der Wechselwirkung im Kontakt während der ebenen Kollision. Tatsächlich zerfällt das Kontaktgebiet bei der tangentialen Belastung im Allgemeinen in ein inneres Haft- und ein äußeres Gleitgebiet. Das folgende Unterkapitel widmet sich daher elastischen ebenen Stößen unter der Berücksichtigung des lokalen Gleitens im Kontakt. Es werden elastisch homogene und inhomogene Medien behandelt. Da das geschilderte kontaktmechanische Modell des Stoßproblems mit Reibung den Anspruch hat, prädiktive Kraft zu besitzen, werden die theoretischen Vorhersagen außerdem mit experimentellen Ergebnissen verglichen. 


\subsubsection{Homogene Medien}

Das schiefe Stoßproblem von Kugeln mit einem endlichen Reibkoeffizienten $\mu$, d. h. unter Berücksichtigung von lokalem Gleiten im Kontakt, kann man selbst für elastische, homogene Medien nicht mehr analytisch lösen. Das Problem besteht dabei in der kontaktmechanisch korrekten Bestimmung der Tangentialkraft, wofür allerdings unterschiedliche numerische Methoden zur Verfügung stehen. Maw et al. [4] beschrieben und implementierten ein numerisches Verfahren, um den Regelsatz von Mindlin und Deresiewicz [7] für elastische Tangentialkontakte mit beliebigen Belastungsgeschichten auf das schiefe Stoßproblem anzuwenden. Sie stellten fest, dass das Stoßproblem, geschrieben in geeigneten dimensionslosen Größen, nur von den beiden Parametern

$$
\chi:=\frac{l}{2 \kappa}, \quad \psi:=\frac{l \tan \alpha}{\mu}
$$

abhängt. Dabei ist $\chi$ bereits aus der Beschreibung des analogen Problems ohne Gleiten bekannt; $\psi$ charakterisiert die Reibeigenschaften des Systems, der (generalisierte) Stoßwinkel $\alpha$ ist in Gl. (2.58) definiert. Maw et al. bestimmten numerisch unter anderem verschiedene Zeitverläufe der Tangentialkraft in Abhängigkeit der beiden obigen Parameter. In NachfolgePublikationen [8,9] verglichen sie außerdem sehr erfolgreich die Vorhersagen ihres Modells mit eigenen Experimenten für den verallgemeinerten Rückprallwinkel als Funktion von $\psi$. Jäger [10] implementierte seine allgemeine Lösung des Tangentialkontaktproblems mithilfe der Superposition von Cattaneo-Mindlin-Lösungen, um das Stoßproblem zu lösen. Von Willert und Popov [11] stammt eine äquivalente aber einfachere Implementierung im Rahmen der MDR.

Aus der im dritten Kapitel gezeigten Lösung des Tangentialkontaktproblems von Kugeln folgt, dass der Stoß für

$$
l v_{x, K, 0}>\mu\left|v_{z, K, 0}\right| \Leftrightarrow \psi>1
$$

im Regime vollständigen Gleitens beginnt. Während des vollständigen Gleitens ist

$$
\left|F_{x}\right|=\mu\left|F_{z}\right|
$$

und damit wegen der Bewegungsgleichung (6.1)

$$
v_{x, K}=v_{x, K, 0}+\frac{\mu}{\kappa}\left(v_{z, K}-v_{z, K, 0}\right) \text {. }
$$

Daraus folgt, dass der gesamte Stoß im Regime vollständigen Gleitens verläuft, falls

$$
v_{x, K, e}=v_{x, K, 0}+\frac{\mu}{\kappa}\left(v_{z, K, e}-v_{z, K, 0}\right)>\frac{\mu v_{z, K, e}}{l} \Leftrightarrow \psi>\psi_{c}:=2 \chi\left(1+\epsilon_{z}\right)-\epsilon_{z} .
$$

Für elastische Stöße ist natürlich $\epsilon_{z}=1$, die Beziehung ist aber auch für inelastische Stöße mit $\epsilon_{z}<1$ korrekt [6], daher wurde die etwas allgemeinere Formulierung gewählt. Im 
Fall vollständigen Gleitens während des ganzen Stoßvorgangs ist die tangentiale Stoßzahl elementarerweise durch

$$
\epsilon_{x}^{\mathrm{fs}}=\frac{2 \chi}{\psi}\left(1+\epsilon_{z}\right)-1
$$

gegeben. Die Ergebnisse (6.56) und (6.58) ergeben sich allein aus der makroskopischen Dynamik und dem Reibgesetz in globaler Form, von der konkreten kontaktmechanischen Wechselwirkung hängen sie ansonsten nicht ab.

Die allgemeine Lösung (mit partiellem Gleiten und Haften) für $\epsilon_{x}$ als Funktion von $\chi$ und $\psi$ ist als Konturlinien-Diagramm in Abb. 6.7 gezeigt. Offenbar hängt die tangentiale Stoßzahl für $\psi<1$ und kleine Werte von $\chi$ nur schwach von $\psi$, also dem Reibungskoeffizienten und dem Stoßwinkel, ab.

Abb. 6.8 zeigt den Verlauf der normierten Tangentialkraft während des Stoßes für $\chi=1,44$ und verschiedene Werte von $\psi$ (nach [4]). Man erkennt die unterschiedlichen Ablösepunkte von dem Verlauf bei vollständigem Gleiten.

Abb.6.7 KonturlinienDiagramm der tangentialen Stoßzahl für den elastischen Stoß mit Gleiten als Funktion der Parameter $\chi$ und $\psi$. Die durchgezogenen Linien bezeichnen die in den Gl. (6.54) und (6.57) definierten Bereichsgrenzen

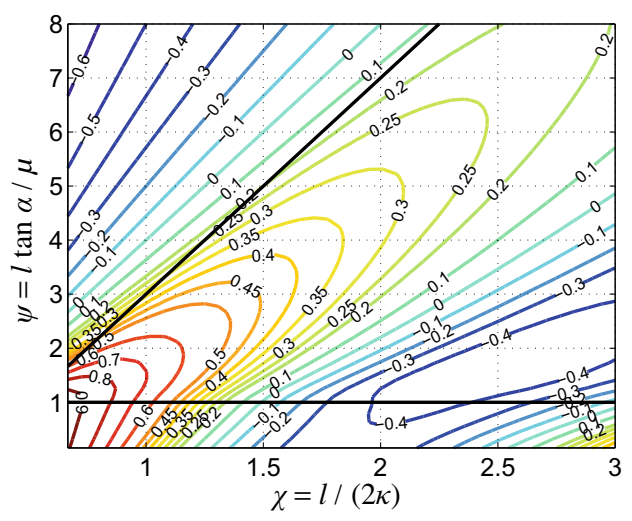

Abb.6.8 Verlauf der normierten Tangentialkraft während des elastischen schiefen Stoßes einer vollständig homogenen Kugel mit $v=0,3$ (daraus folgt $\chi \approx 1,44$ ) für verschiedene Werte des generalisierten Stoßwinkels $\psi$ (nach [4])

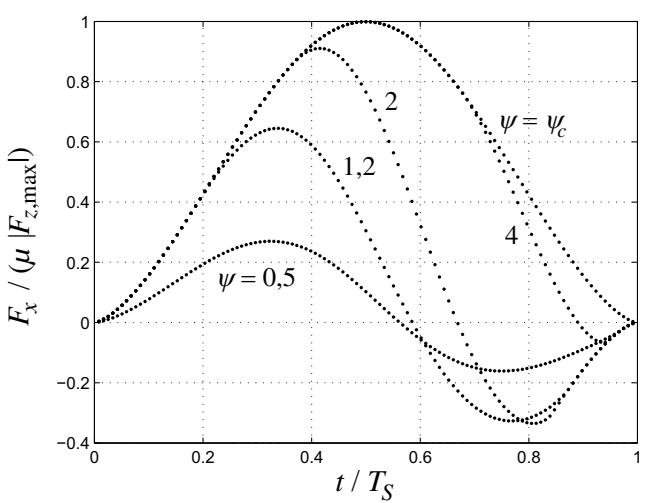




\section{Verteilung der Energiedissipation im Kontaktgebiet}

Die Energiedissipation durch Reibung ist in der Regel mit dem Verschleiß der beteiligten Oberflächen verbunden. Im Fall des ebenen Stoßes mit Gleiten spricht man dabei von „Schlagverschleiß“, der im Unterkapitel 8.1 beschrieben ist. Unter Umständen ist dabei nicht nur die Stoßzahl - und damit die gesamte während des Stoßes dissipierte Energie von Interesse, sondern auch die Verteilung der Dissipation im Kontaktgebiet. Dieser Aspekt wurde in der Arbeit [12] untersucht.

Im Rahmen des oben geschilderten Modells für den ebenen elastischen Stoß mit Reibung ist klar, dass Energie zu jedem Zeitpunkt nur im Gleitgebiet dissipiert wird. Die lokale Reibleistung pro Fläche, $\dot{w}$, beträgt daher

$$
\dot{w}(r, t)=\frac{2 \mu \tilde{E}}{\pi \tilde{R}} \sqrt{a(t)^{2}-r^{2}} v_{\mathrm{rel}}(r, t), \quad c(t)<r \leq a(t) .
$$

Dabei bezeichnet $v_{\text {rel }}$ die (lokale) relative Geschwindigkeit zwischen den kontaktierenden Oberflächen, die am einfachsten mithilfe der zu Gl.(4.8) analogen Abel-Transformation

$$
v_{\mathrm{rel}}(r, t)=\frac{2}{\pi} \int_{c(t)}^{r} \frac{v_{\mathrm{rel}}^{1 D}(x, t)}{\sqrt{r^{2}-x^{2}}} \mathrm{~d} x
$$

aus der Relativgeschwindigkeit im MDR-Modell des Stoßproblems bestimmbar ist. Bei der numerischen Ausführung dieser Transformation ist es hilfreich, von der partiellen Integration

$$
v_{\mathrm{rel}}(r, t)=v_{\mathrm{rel}}^{1 D}(r, t)-\frac{2}{\pi} \int_{c(t)}^{r} \arcsin \left(\frac{x}{r}\right) \frac{\mathrm{d}}{\mathrm{d} x}\left(v_{\mathrm{rel}}^{1 D}(x, t)\right) \mathrm{d} x
$$

Gebrauch zu machen, um die Singularität des Integranden an der Stelle $x=r$ zu vermeiden [13].

Die gesamte lokale, durch Reibung während der Kollision dissipierte Energie pro Fläche, $w$, normiert auf den mittleren Wert

$$
w_{0}:=\frac{\left|\Delta U_{\mathrm{kin}}\right|}{\pi a_{\max }^{2}}=\frac{\tilde{m} \kappa}{2 \pi a_{\max }^{2}} v_{x, K, 0}^{2}\left(1-\epsilon_{x}^{2}\right),
$$

hängt im Allgemeinen von den beiden Stoßparametern $\chi$ und $\psi$ ab. Abb. 6.9 zeigt die Verteilung der Energiedissipation über das Kontaktgebiet während des Stoßes in normierten Größen für $\chi=1,4$ und verschiedene Werte von $\psi$. Offensichtlich ist die Dissipation für kleine Werte von $\psi$ - also Dominanz des Haftregimes - am Rand des Kontaktgebiets konzentriert, während bei Dominanz des Gleitens mehr Energie im Zentrum des Kontaktgebiets verloren geht. 
Abb.6.9 Verteilung der normierten dissipierten Energie pro Fläche als Funktion der normierten radialen Koordinate für $\chi=1,4$ und verschiedene Werte von $\psi$ [12]. Die dünne durchgezogene Linie bezeichnet den Fall vollständigen Gleitens

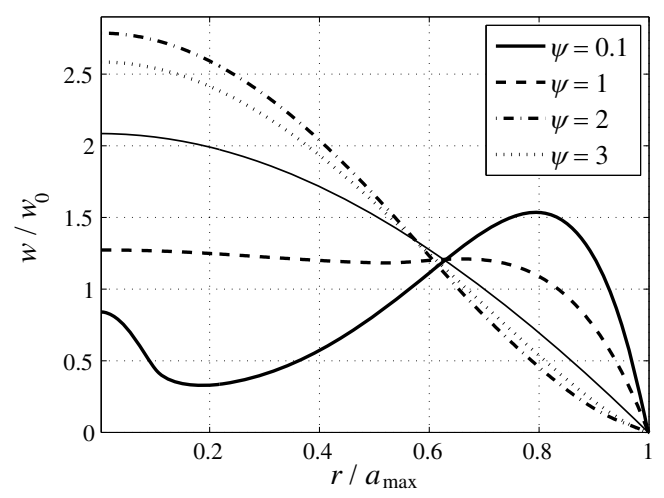

Je näher die Stoßkonfiguration zu Parameterkombinationen liegt, bei denen der Kontakt während der ganzen Kollision vollständig gleitet, desto schwächer ist die explizite Abhängigkeit der Dissipationsverteilung von den Stoßparametern. Abb. 6.10 zeigt die Verteilung in normierten Größen für verschiedene Werte von $\chi$ und $\psi=\psi_{c} / 3$ sowie $\psi=\psi_{c} / 5$; dabei bezeichnet $\psi_{c}$ den in Gl.(6.57) gegebenen kritischen Wert, bei dem der Kontakt während der ganzen Kollision vollständig gleitet.

Falls $\psi \geq \psi_{c}$, ist die Verteilung der Dissipation, in normierten Größen, sogar unabhängig von $\psi$ und $\chi$ und kann durch den analytischen Ausdruck [12]

$$
\frac{w^{\mathrm{fs}}}{w_{0}} \approx 2,1208-0,2488 \frac{r}{a_{\max }}-1,9093\left(\frac{r}{a_{\max }}\right)^{2}, \quad r \leq a_{\max },
$$

approximiert werden.

\section{Starrkörpermodelle des Stoßes mit Gleiten}

Wegen der Komplexität der kontaktmechanischen Behandlung von Stößen mit Reibung wird teilweise die Elastizität vernachlässigt und nur der Zusammenstoß starrer Körper betrachtet.

Abb.6.10 Verteilung der normierten dissipierten Energie pro Fläche als Funktion der normierten radialen Koordinate für verschiedene Werte $\chi[12]$. Die roten Kurven gehören zu $\psi=(4 \chi-1) / 3$, die schwarzen zu $\psi=(4 \chi-1) / 5$

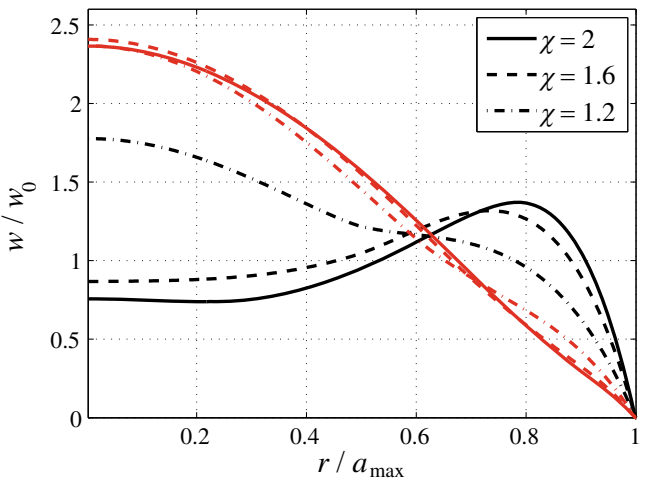


Der Stoß selbst ist in diesen Starrkörper-Modellen eine Unstetigkeit der Bewegung, die durch Kraftstöße oder Stoßzahlen beschrieben wird. Letztere muss man aus rein makroskopischen Erwägungen (Erhaltungssätze, Kinematik) bestimmen. Für den schiefen Stoß mit Gleiten gibt es dann genau zwei verschiedene Regime: Gleiten im (buchstäblichen) Kontaktpunkt oder stationäres Abrollen. Im ersten Fall ist die Stoßzahl durch Gl. (6.58) gegeben, Abrollen ist im Starrkörper-Modell durch die Bedingung $\epsilon_{x}=0$ charakterisiert. Doménech-Carbó [14] definiert den Übergang zwischen beiden Regimen durch die Bedingung

$$
\epsilon_{x}^{\mathrm{fs}}=0 \quad \Leftrightarrow \quad \psi_{\text {trans }}=2 \chi\left(1+\epsilon_{z}\right)
$$

Alternativ kann man mit Gl.(6.56) den Zeitpunkt bestimmen, wann der Kontaktpunkt bei Gleiten die Geschwindigkeit Null erreicht [15]. Danach geht im Starrkörper-Modell der Kontakt in das Regime des stationären reinen Abrollens über und die Tangentialkraft im Kontakt verschwindet.

Starrkörper-Modelle sind sehr einfach zu benutzen. Allerdings führen sie zu Widersprüchen, wie schon bei der Darstellung der „elementaren“ Lösung des Stoßproblems ohne Gleiten angemerkt wurde, und - abseits des Regimes vollständigen Gleitens - zu deutlichen Abweichungen bei der bestimmten Stoßzahl von Modellen, die die Elastizität der Kontaktpartner berücksichtigen, man vergleiche beispielsweise mit Abb. 6.7.

\section{Vollständig linearer Kontakt}

Eine weitere Möglichkeit zur Vereinfachung des Problems besteht darin, den Kontakt durch eine einzelne reibbehaftete lineare Feder mit den Steifigkeiten $k_{z}$ und $k_{x}$ zu modellieren (oder, mit anderen Worten, als Flachstempelkontakt zu betrachten). Das Kraftgesetz für die Tangentialkraft lautet dann

$$
\begin{aligned}
& \dot{F}_{x}=k_{x} v_{x, K}, \quad \text { falls }\left|F_{x}\right| \leq \mu\left|F_{z}\right|, \\
& F_{x}=\mu\left|F_{z}\right| \operatorname{sgn}\left(v_{x, K}\right), \quad \text { sonst. }
\end{aligned}
$$

Das entstehende abschnittsweise lineare Bewegungsgleichungssystem kann theoretisch analytisch gelöst werden, indem man sorgfältig Phasen von Haften und Gleiten der Feder während des Kontaktes verfolgt; allerdings ist eine numerische Lösung sicherlich vorzuziehen. Man stellt fest, dass das Problem in dimensionsfreier Formulierung ebenfalls nur von den Parametern $\psi$ und $\chi$ abhängt, wobei $l=k_{x} / k_{z}$. Die Stoßzahl als Funktion dieser beiden Parameter ist in Abb. 6.11 gezeigt. Offenbar unterscheidet sich die Lösung nur in Bereichen von großen $\chi$ und kleinen $\psi$ (also bei Dominanz des Haft-Regimes) merklich von der in Abb. 6.8 dargestellten nicht-linearen Lösung. Insbesondere ist durch die Herleitung der in den Gl.(6.54) und (6.57) definierten Bereichsgrenzen klar, dass diese auch für das lineare Modell gültig sind.

Ein ausführlicher Vergleich des linearen und nicht-linearen Modells (einschließlich eines semi-rigorosen Modells auf Grundlage der Cattaneo-Mindlin-Lösung, d.h. ohne Berücksichtigung von Gedächtnis-Effekten des Tangentialkontaktproblems) kann bei Thornton et al. [16] nachgeschlagen werden. 
Abb.6.11 KonturlinienDiagramm der tangentialen Stoßzahl für den elastischen Stoß mit Gleiten als Funktion der Parameter $\chi$ und $\psi$, wenn der Kontakt als eine einzelne lineare Feder modelliert wird. Die durchgezogenen Linien bezeichnen die im Text erläuterten Bereichsgrenzen

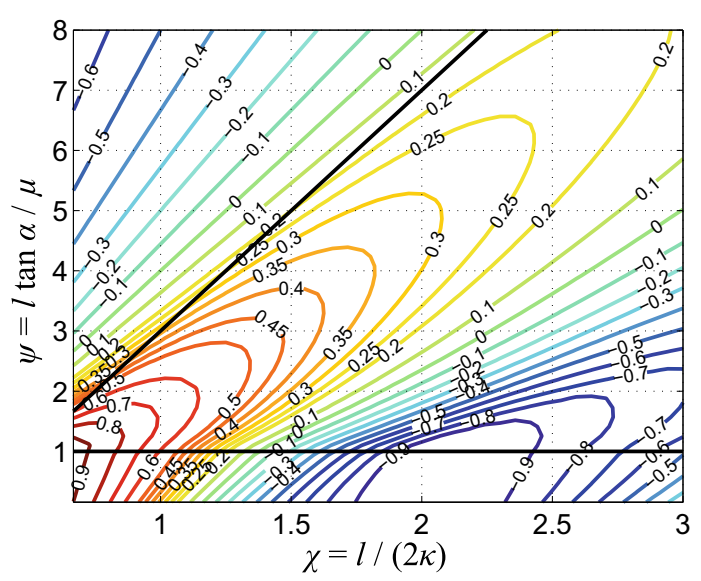

\subsubsection{Funktionale Gradientenmedien}

Mithilfe der im dritten Kapitel geschilderten Lösung für den Tangentialkontakt von Kugeln mit einer elastischen Gradierung in der Form eines Potenzgesetzes mit dem Exponenten $k$ (beziehungsweise mithilfe der Deutung dieser Lösung im Rahmen der MDR) kann man das entsprechende schiefe Stoßproblem mit Gleiten in völliger Analogie zu dem im vorherigen Abschnitt dargestellten homogenen Fall numerisch lösen [17]. Die tangentiale Stoßzahl hängt nur von den Parametern

$$
k, \quad \chi=\frac{l}{2 \kappa}, \quad \psi=\frac{l \tan \alpha}{\mu}
$$

ab, wobei man für das Mindlin-Verhältnis $l$ den Wert des Gradientenmediums verwenden muss. Die explizite Abhängigkeit von $k$ ist allerdings sehr schwach. Das kann man wie folgt verstehen: Auch für den Stoß mit Gleiten ist - wie man durch numerische Rechnungen zeigen kann - für die tangentiale Stoßzahl die Änderung des Exponenten der elastischen Gradierung über Gl. (5.32) äquivalent zu einer bestimmten Änderung des Exponenten des Indenterprofils ${ }^{4}$. Letzteres hat aber, wie im letzten Abschnitt demonstriert, in großen Parameterbereichen nur einen geringen Einfluss auf die Stoßzahl; das gleiche gilt daher auch für die elastische Inhomogenität. Zur Bestimmung der Stoßzahl kann man daher in guter Näherung die in Abb. 6.7 gezeigte homogene Lösung heranziehen.

In der Arbeit [17] wurden nur kleine Werte von $\chi$ berücksichtigt und daher fälschlicherweise geschlussfolgert, dass die Stoßzahl gar keine explizite Abhängigkeit von $k$ aufweist. Dies ist, wie beschrieben, im Allgemeinen nicht korrekt.

\footnotetext{
${ }^{4}$ Die makroskopische Dynamik bleibe wiederum von dieser Profiländerung unbeeinflusst, siehe die Betrachtung nicht-parabolischer Indenterformen in Abschn. 6.1.1.
} 


\subsubsection{Vergleich mit experimentellen Ergebnissen}

Die experimentelle Literatur zu elastischen schiefen Stößen von Kugeln ist nicht sehr umfangreich aber dafür substanziell.

Foerster et al. [18] untersuchten binäre Kollisionen von kleinen Glaskugeln $(R=$ $3,18 \mathrm{~mm}$ ) und Stöße einer kleinen Glaskugel auf eine massive Aluminiumplatte. Wegen der hohen Inelastizität der Stöße zwischen Kugel und Platte $\left(\epsilon_{z} \approx 0,83\right)$ werden an dieser Stelle nur die binären Kollisionen $\left(\epsilon_{z} \approx 0,97\right)$ berücksichtigt. Die Autor*innen bestimmten unter anderem Verläufe für den Rückprallwinkel des Kontaktpunktes $\alpha^{*}$ als Funktion des Einfallswinkels $\alpha$. Für den Reibbeiwert und die Querkontraktionszahl gaben sie $\mu=0,092$ sowie $v=0,22$ an, daraus folgt $\chi \approx 1,53$.

Gorham und Kharaz [19] unternahmen ausführliche experimentelle Studien zu Stößen von Aluminiumoxid-Kugeln $(R=2,5 \mathrm{~mm})$ auf eine Glasplatte. Die Kollisionen waren annähernd ideal-elastisch $(\epsilon \approx 0,98)$. Da die beteiligten Materialien elastisch nicht ähnlich sind, ist die Bestimmung von $\chi$ nicht elementar, die Autor*innen selbst gaben $\chi=1,5$ sowie $\mu=0,092$ an. Sie bestimmten unter anderem Verläufe für $\alpha^{*}$ und die Stoßzahl des Schwerpunktes, $\epsilon_{x, S}$, als Funktionen von $\alpha$ und verglichen ihre Ergebnisse bereits teilweise mit der kontaktmechanischen Lösung von Maw et al. [4].

Dong und Moys [20] untersuchten Stöße von Stahl-Kugeln $(R=22,225 \mathrm{~mm})$ auf einen massiven Stahl-Block. Die Inelastizität der Kollisionen war relativ hoch $\left(\epsilon_{z} \approx 0,9\right)$, die Autor*innen gaben außerdem $v=0,3$ (daraus folgt $\chi \approx 1,44$ ) sowie $\mu=0,091$ an und bestimmten unter anderem Verläufe für $\alpha^{*}$ und $\epsilon_{x, S}$ als Funktionen von $\alpha$. Sie betrachteten außerdem den Einfluss einer bereits vor der Kollision vorhandenen Rotation der Kugel.

Abb. 6.12 zeigt einen Vergleich zwischen den experimentellen Ergebnissen und der Vorhersage des kontaktmechanischen Modells (elastisch, homogen, mit Gleiten) für die Abhängigkeit des Rückprallwinkels des Kontaktpunktes $\alpha^{*}$ als Funktion des Einfallswinkels $\alpha$. Da die Werte von $\chi$ und $\mu$ bei allen Experimenten sehr nah beieinander liegen, ist nur eine

Abb.6.12 Rückprallwinkel (in Grad) als Funktion des Einfallswinkels (in Grad) für den schiefen Stoß von Kugeln mit Gleiten. Experimentelle Ergebnisse und numerische Lösung des kontaktmechanischen Modells für $\chi=1,5$ und $\mu=0,092$ (durchgezogene Linie)

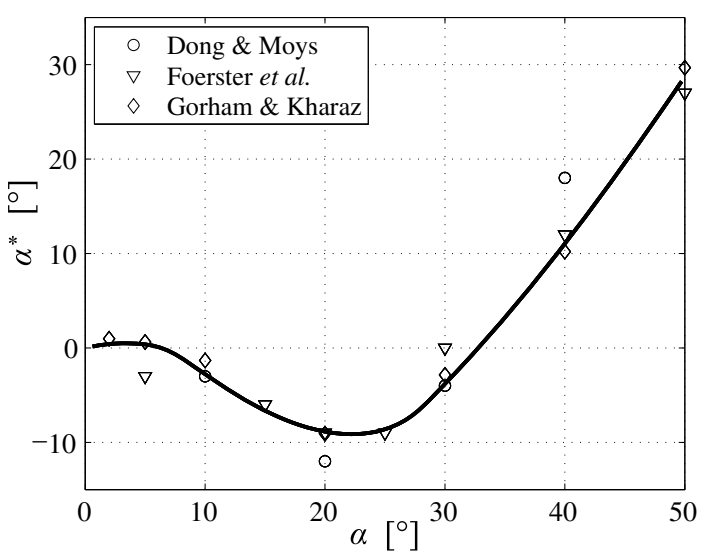


Abb.6.13 Tangentiale

Stoßzahl des Schwerpunkts

(ohne ursprüngliche Rotation)

als Funktion des

Einfallswinkels (in Grad) für den schiefen Stoß einer Kugel auf eine Platte. Experimentelle

Ergebnisse und numerische

Lösung des kontakt-

mechanischen Modells für

$\chi=1,5$ und $\mu=0,092$

(durchgezogene Linie)

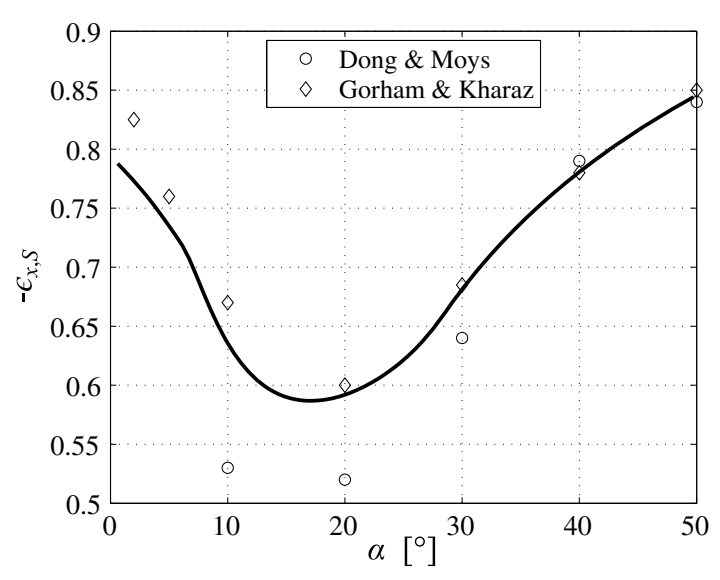

einzige theoretische Kurve (mit $\chi=1,5$ und $\mu=0,092$ ) in dem Diagramm gezeigt. Es sind außerdem nur Werte bis $\alpha=50^{\circ}$ dargestellt, da der Kontakt bereits bei $\alpha \approx 30^{\circ}$ während der gesamten Kollision vollständig gleitet. Die theoretische Vorhersage stimmt gut mit den Experimenten überein. Dabei muss man berücksichtigen, dass in dem kontaktmechanischen Modell kein freier Parameter zur „Optimierung“ der theoretischen Vorhersage vorhanden ist. Besonders gut ist die Übereinstimmung mit den Ergebnissen von Gorham \& Kharaz; diese weisen auch die geringste Streuung der Messwerte in allen genannten Publikationen auf.

Abb. 6.13 zeigt einen Vergleich zwischen Theorie und Experiment für die tangentiale Stoßzahl des Schwerpunktes als Funktion des Einfallswinkels für den Stoß einer Kugel auf eine Platte, wenn die Kugel vor der Kollision nicht rotiert. Die Übereinstimmung ist wiederum für die experimentellen Ergebnisse von Gorham \& Kharaz gut; der Vergleich mit den Resultaten von Dong \& Moys ist dagegen weniger zufriedenstellend, was man unter anderem auf die Inelastizität der Kollisionen zurückführen kann.

\subsection{Viskoelastischer schiefer Stoß mit Gleiten}

Wie für den Fall ohne Gleiten demonstriert, spielt die konkrete viskoelastische Rheologie für das tangentiale Stoßproblem nur eine untergeordnete Rolle, wenn die Materialeigenschaften durch die normale Stoßzahl charakterisiert werden. Es ist daher in vielen Fällen ausreichend, das einfachste rheologische Modell, den Kelvin-Voigt-Körper, zu betrachten.

In der Literatur finden sich nur wenige systematische Untersuchungen des viskoelastischen ebenen Stoßes mit Gleiten. Rad und Pishkenari [21] verwendeten ein makroskopisches Starrkörper-Modell der Reibung, dessen Beschränkungen bereits im vorherigen Unterkapitel anhand des elastischen Problems diskutiert wurden. Brilliantov et al. [22] und später Schwager et al. [23] beschrieben die Reibung mithilfe eines tribologischen Modells, das auf Cundall 
und Strack [24] zurückgeht, und in dem die tangentiale Kraft mithilfe einer durch das Reibgesetz beschränkten linearen Feder bestimmt wird. Während dies im elastischen Fall auf die Lösung des Flachstempel-Problems hinausläuft - dessen Lösung sich, wie gezeigt, in großen Parameterbereichen nicht wesentlich von der des parabolischen Problems unterscheidet geht im viskoelastischen Fall dadurch eine relevante Quelle der Energie-Dissipation verloren, nämlich der viskose Anteil der Tangentialspannungen. Kontaktmechanisch rigorose Lösungen des Problems gaben Kusche [25] und Willert et al. [6].

Im Rahmen der Cattaneo-Mindlin-Näherung kann man das Problem exakt durch die MDR lösen. Wenn das Normalstoßproblem gelöst wurde, bereitet die Untersuchung der Tangentialbewegung innerhalb der MDR keine größeren Schwierigkeiten. Die tangentiale Streckenlast der viskoelastischen Bettung,

$$
q_{x}(x, t)=\frac{8}{3}\left(G u_{x}(x, t)+\eta \dot{u}_{x}(x, t)\right),
$$

muss das in den Gl. (4.28) und (4.29) gegebene lokale Amontons-Coulomb-Reibgesetz erfüllen. Wenn das entsprechende Element haften kann, sind die Werte von $\mathrm{d} u_{x}$ und $\dot{u}_{x}$ durch die Bewegung des Eindruckkörpers vorgegeben. Wenn das Element gleitet, ist durch das Reibgesetz der Wert von $q_{x}$ bekannt und Gl.(6.68) liefert eine Bestimmungsgleichung für $u_{x}$. Durch Integration der Streckenlast ergibt sich die gesamte tangentiale Kraft, mit der die Bewegungsgleichung (6.1) gelöst wird. Die tangentiale Stoßzahl hängt nur von den Parametern

$$
\chi=\frac{1}{3 \kappa}, \quad \psi=\frac{2 \tan \alpha}{3 \mu}
$$

sowie der normalen Stoßzahl $\epsilon_{z}$ ab. Da keine dieser drei Größen in ihrem Einfluss vernachlässigbar (oder trivial) ist und eine übersichtliche kompakte Darstellung der vollständigen Lösung $\epsilon_{x}=\epsilon_{x}\left(\chi, \psi, \epsilon_{z}\right)$ in einer einzelnen Abbildung kaum möglich erscheint, ist im Anhang eine einfache Implementierung des MDR-Modells zur Lösung des Stoßproblems in der Programmiersprache der kommerziellen Software MATLAB des Unternehmens MathWorks ${ }^{\circledR}$ gegeben, die man zur Lösung für eine beliebige Parameterkombination heranziehen kann ${ }^{5}$. Abb. 6.14 zeigt außerdem die Lösung für den sicher wichtigsten Fall einer homogenen Kugel (und damit $\kappa=2 / 7$ ) als Konturliniendiagramm in Abhängigkeit der verbleibenden Einflussgrößen $\psi$ und $\epsilon_{z}$. Interessanterweise hängt die tangentiale Stoßzahl für kleine Werte von $\epsilon_{z}$ außerhalb des Bereichs vollständigen Gleitens nur sehr schwach von $\psi \mathrm{ab}$.

\footnotetext{
${ }^{5}$ Dies dient darüber hinaus zur Veranschaulichung der Tatsache, dass die numerische Implementierung der Regeln der MDR eine elementar einfache Aufgabe darstellt.
} 


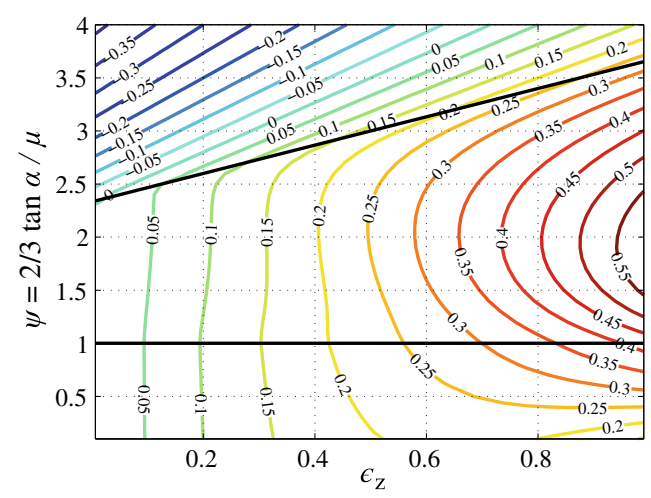

Abb.6.14 Konturlinien-Diagramm der tangentialen Stoßzahl für den ebenen Stoß mit Gleiten einer homogenen Kugel auf ein inkompressibles Kelvin-Voigt-Medium als Funktion des Parameters $\psi$ und der normalen Stoßzahl. Die durchgezogenen Linien bezeichnen die in den Gl. (6.54) und (6.57) definierten Bereichsgrenzen

\subsection{Elasto-Plastischer schiefer Stoß mit Gleiten}

Wie im Kapitel zum reinen Normalstoß ausgeführt, können auch bereits bei kleinen Stoßgeschwindigkeiten plastische Deformationen in den kollidierenden Körpern auftreten. Das entsprechende ebene Kollisionsproblem unter Berücksichtigung der Plastizität und der Reibung ist besonders für den Schlagverschleiß und die Erosion metallischer Oberflächen relevant. Wegen der großen Komplexität des Kontaktproblems gibt es nur wenige, in der Umsetzung aufwendige Modelle zur rigorosen Behandlung des elasto-plastischen ebenen Stoßes mit Reibung.

Wu et al. [26] konstruierten ein FEM-basiertes elastisches und elasto-plastisches Modell, das auf Oberflächenelementen beruht, die ein lokales Amontons-Coulomb-Reibgesetz reproduzieren. Die Autor*innen verglichen ihre Vorhersagen mit Versuchsergebnissen von Gorham und Kharaz [19] - für den schiefen Stoß einer Aluminiumoxid-Kugel auf eine dicke Platte aus einer Aluminium-Legierung - und erzielten dabei eine gute Übereinstimmung zwischen Theorie und Experiment.

Brake [27] publizierte eine semi-analytische Verallgemeinerung des elastischen Modells von Maw et al. [4] und verglich die theoretischen Vorhersagen ebenfalls erfolgreich mit den Experimenten von Gorham \& Kharaz. Für das Normalkontaktproblem verwendete er eine verbesserte Version des Interpolationsmodells, bei dem die Kontaktkraft im elastoplastischen Bereich in einen elastischen und einen plastischen Anteil zerlegt wird. Der Tangentialkontakt wird im Rahmen der Cattaneo-Mindlin-Näherung behandelt. Dazu führte Brake für die Tangentialspannungen in einem Ring mit Radius $r$ den gleichen Interpolationsansatz ein, wie für die Normalkraft. Anschließend bestimmte er die elastischen Verschiebungen $u_{x}$, die aus den superponierten (elastischen und plastischen) Tangentialspannungen 
resultieren; schließlich wird iterativ der Radius $c$ des Haftgebiets bestimmt, sodass die gemischten Randbedingungen im Haft- und Gleitgebiet erfüllt sind.

Einfache robuste Modelle für den allgemeinen ebenen elasto-plastischen Stoß mit Reibung gibt es leider nicht. Für sehr flache Stoßwinkel, wenn der Kontakt während der gesamten Kollision vollständig gleitet, kann man allerdings die elementare Full-Slip-Lösung aus Gl. (6.58) verwenden.

\subsection{Zusammenfassung}

Wegen des komplexen Wechselspiels zwischen der Kontaktmechanik und der makroskopischen Dynamik kann der ebene Stoß von Kugeln mit Reibung im Allgemeinen nicht geschlossen analytisch behandelt werden. Für den elastischen Fall ohne lokales Gleiten (also mit einem theoretisch unendlich großen Reibbeiwert) kann man allerdings mithilfe Hypergeometrischer Funktionen eine analytische Lösung finden. In Ausnahmefällen kann dieser Stoß (elastisch, ohne Gleiten) sogar ohne Energie-Dissipation ablaufen.

Eine elastische Inhomogenität des Mediums hat nur einen geringen Einfluss auf die tangentiale Stoßzahl, solange das Mindlin-Verhältnis aus tangentialer und normaler Steifigkeit des Flachstempel-Kontaktes eine Konstante ist. Das Profil der kollidierenden Körper in der Nähe des Kontaktes ist für das Stoßproblem bei vielen Parameterkombinationen ebenfalls von untergeordneter Bedeutung (solange die makroskopische Bewegungsgleichung unverändert bleibt).

Die elastische (oder viskoelastische) Kollision mit Gleiten kann man numerisch besonders einfach im Rahmen der MDR untersuchen. Die tangentiale Stoßzahl hängt im elastischen Fall nur von zwei dimensionsfreien Parametern ab, von denen der eine die geometrischen und der andere die Reibeigenschaften des Systems charakterisiert. Für ausreichend flache Stoßwinkel wird der Kontakt während des gesamten Stoßes vollständig gleiten. Die Lösung des Kontakt- (und damit auch des Stoßproblems) ist in diesem Fall trivial, da die Reibkraft durch das Amontons-Coulomb-Gesetz in seiner makroskopischen Form bekannt ist. In Konfigurationen mit nur lokalem Gleiten ist das Verhalten des Systems dagegen komplizierter.

Bei Dominanz des Haft-Regimes wird die meiste Reibungsenergie am Rand des maximalen Kontaktgebiets während der Kollision dissipiert. Bei ausgeprägtem (oder vollständigem) Gleiten während des Stoßes ist dagegen das Zentrum des Kontaktgebiets die Hauptquelle des Energieverlustes.

Starrkörpermodelle versuchen, die Reibkraft ausschließlich durch makroskopische Erwägungen zu bestimmen. Da es makroskopisch aber nur zwei Konfigurationen des Kontaktpunktes gibt (Haften oder Gleiten), betrachten Starrkörpermodelle nur die Fälle des globalen Gleitens und des reinen Rollens. Dies steht im Widerspruch zur notwendigen Elastizität des Kontaktes und führt abseits des Regimes des vollständigen Gleitens zu deutlichen Fehlern der Modellierung. 
In viskoelastischen ebenen Stößen mit Gleiten spielt die konkrete viskoelastische Rheologie für die Lösung des Stoßproblems nur eine geringe Rolle; es ist daher in der Regel ausreichend, zur Charakterisierung des Einflusses der Viskoelastizität die normale Stoßzahl zu verwenden.

Für elasto-plastische ebene Kollisionen mit Reibung existieren nur wenige, in der Anwendung aufwendige, semi-analytische oder vollständig numerische Modelle.

\section{Literatur}

1. Gross, D., Hauger, W., Schröder, J., \& Wall, W. A. (2012). Technische Mechanik 3: Kinetik (12. Aufl.). Berlin: Springer Vieweg.

2. Barber, J. R. (1979). Adhesive contact during the oblique impact of elastic spheres. ZAMP Zeitschrift für angewandte Mathematik und Physik, 30(3), 468-476.

3. Jäger, J. (1994). Analytical solutions of contact impact problems. Applied Mechanics Review, 47(2), 35-54.

4. Maw, N., Barber, J. R., \& Fawcett, J. N. (1976). The oblique impact of elastic spheres. Wear, $38(1), 101114$.

5. Lyashenko, I. A., \& Popov, V. L. (2015). Impact of an elastic sphere with an elastic half space revisited: numerical analysis based on the method of dimensionality reduction. Scientific Reports, 5, 8479. https://doi.org/10.1038/srep08479.

6. Willert, E., Kusche, S., \& Popov, V. L. (2017). The influence if viscoelasticity on velocitydependent restitutions in the oblique impact of spheres. Facta Universitatis, Series Mechanical Engineering, 15(2), 269-284.

7. Mindlin, R. D., \& Deresiewicz, H. (1953). Elastic spheres in contact under varying oblique forces. Journal of Applied Mechanics, 20(3), 327-344.

8. Maw, N., Barber, J. R., \& Fawcett, J. N. (1977). The rebound of elastic bodies in oblique impact. Mechanics Research Communications, 4(1), 17-22.

9. Maw, N., Barber, J. R., \& Fawcett, J. N. (1981). The role of elastic tangential compliance in oblique impact. Journal of Lubrication Technology, 103(1), 74-80.

10. Jäger, J. (1992). Elastic impact with friction. Dissertation. TU Delft (1992)

11. Willert, E., \& Popov, V. L. (2016). (2016) Impact of an elastic sphere with an elastic half space with a constant coefficient of friction: Numerical analysis based on the method of dimensionality reduction. ZAMM Zeitschrift für Angewandte Mathematik und Mechanik, 96(9), 1089-1095.

12. Willert, E. (2019). Energy loss and wear in spherical oblique elastic impacts. Facta Universitatis, Series Mechanical Engineering, 17(1), 75-85.

13. Benad, J. (2018). Fast numerical implementation of the MDR transformations. Facta Universitatis, Series Mechanical Engineering, 16(2), 127-138.

14. Doménech-Carbó, A. (2013). Analysis of oblique rebound using a redefinition of the coefficient of tangential restitution coefficient. Mechanics Research Communications, 54, 35-40.

15. Pishkenari, H. N., Rad, H. K., \& Shad, H. J. (2017). Transformation of sliding motion to rolling during spheres collision. Granular Matter, 19, 70. https://doi.org/10.1007/s10035-017-0755-0.

16. Thornton, C., Cummins, S. J., \& Cleary, P. W. (2011). An investigation of the comparative behaviour of alternative contact forcemodels during elastic collisions. Powder Technology, 210(3), 189-197.

17. Willert, E., \& Popov, V. L. (2017). The oblique impact of a rigid sphere on a power-law graded elastic halfspace. Mechanics of Materials, 109, 82-89. 
18. Foerster, S. F., Louge, M. Y., Chang, H., \& Allia, K. (1994). Measurements of the collision properties of small spheres. Physics of Fluids, 6(3), 1108-1115.

19. Gorham, D. A., \& Kharaz, A. H. (2000). The measurement of particle rebound characteristics. Powder Technology, 112(3), 193-202.

20. Dong, H., \& Moys, M. H. (2006). Experimental study of oblique impacts with initial spin. Powder Technology, 161(1), 22-31.

21. Rad, H. K., \& Pishkenari, H. N. (2018). Frictional viscoelastic basedmodel for spherical particles collision. Granular Matter, 20, 62. https://doi.org/10.1007/s10035-018-0835-9.

22. Brilliantov, N. V., Spahn, F., Hertzsch, J. M., \& Pöschel, T. (1996). Model for collisions in granular gases. Physical Review E, 53(5), 5382-5392.

23. Schwager, T., Becker, V., \& Pöschel, T. (2008). Coefficient of tangential restitution for viscoelastic spheres. The European Physical Journal E: Soft Matter and Biological Physics, 27(1), 107-114.

24. Cundall, P. A., \& Strack, O. D. L. (1979). A discrete numerical model for granular assemblies. Géotechnique, 29(1), 47-65.

25. Kusche, S. (2016) Simulation von Kontaktproblemen bei linearem viskoelastischem Materialverhalten. Dissertation, Technische Universität Berlin.

26. Wu, C. Y., Thornton, C., \& Li, L. Y. (2008). A semi-analytical model for oblique impacts of elastoplastic spheres. Proceedings of the Royal Society of London, Series A, 465, 937-960.

27. Brake, M. R. (2015). An analytical elastic plastic contact model with strain hardening and frictional effects for normal and oblique impacts. International Journal of Solids and Structures, $62,104-123$.

Open Access Dieses Kapitel wird unter der Creative Commons Namensnennung 4.0 International Lizenz (http://creativecommons.org/licenses/by/4.0/deed.de) veröffentlicht, welche die Nutzung, Vervielfältigung, Bearbeitung, Verbreitung und Wiedergabe in jeglichem Medium und Format erlaubt, sofern Sie den/die ursprünglichen Autor(en) und die Quelle ordnungsgemäß nennen, einen Link zur Creative Commons Lizenz beifügen und angeben, ob Änderungen vorgenommen wurden.

Die in diesem Kapitel enthaltenen Bilder und sonstiges Drittmaterial unterliegen ebenfalls der genannten Creative Commons Lizenz, sofern sich aus der Abbildungslegende nichts anderes ergibt. Sofern das betreffende Material nicht unter der genannten Creative Commons Lizenz steht und die betreffende Handlung nicht nach gesetzlichen Vorschriften erlaubt ist, ist für die oben aufgeführten Weiterverwendungen des Materials die Einwilligung des jeweiligen Rechteinhabers einzuholen.

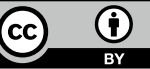

